\title{
Practical approach for the study of metabolic regulation.
}

Macedo, D.V.; Hohl, R; Tessutti, L.S.; Lazarim, F.L.; Silva, F.O.C., Gandra, P.G.; Gadelha, F.; Lourenço, T.F.; Buscariolli, R.; Ferrucci, D.L.; Machado, E.A.; Sarraipa, M.; Lopes, C.R.; Stancanelli, M..

\author{
LABEX, Depto. Bioquímica, IB UNICAMP.
}

First year students in Physical Education must understand metabolic regulation to comprehend the whole integration of biochemical pathways in attempt to establish the relation with exercise. This whole view is not easy to learn and the task becomes even harder with the lack of time at the end of course, when normally the students think about metabolic integration. Trying to get the students attention to this important issue, we developed practical works beginning in the middle of the course, in parallel with theory classes. Blood and urine were collected for metabolite analysis in each practice. The students were divided in groups (10 students) and they created the protocols in form that they only have been guided and directed by the teacher and monitors. The practical activities and biochemical analysis were: six $30 \mathrm{~m}$ sprints with different recovery times (blood lactate and mean velocities), lactate removal from muscle to blood after high intensity exercise (blood lactate), anaerobic threshold (blood lactate and heart rate), the effect of glycogen depletion after high and moderate intensity exercises (plasma glucose and urea concentrations) and low carbohydrate diet vs. normal diet (plasma glucose and urine ketone bodies). After data collection, discussion and interpretation, the students presented orally each work in the same order above. Each presentation had the focus on the metabolic pathways involved in each practice. Group 1: phosphocreatine utilization and resynthesis. Group 2: anaerobic glycolysis, lactate production and removal. Group 3: transition between anaerobic glycolysis and oxidative metabolism. In attempt to promote the integration between muscle and liverGroup 4: protein catabolism after high intensity exercise with low muscular glycogen concentration (transamination, Cori Cycle and gluconeogenesis). Group 5: liver ketogenesis in low carbohydrate diet. This sequence was intended to promote the comprehension of integrated metabolism. As a final activity, the students showed their results in the form of poster. All activities were part of discipline evaluation. All students approved this practical approach. 